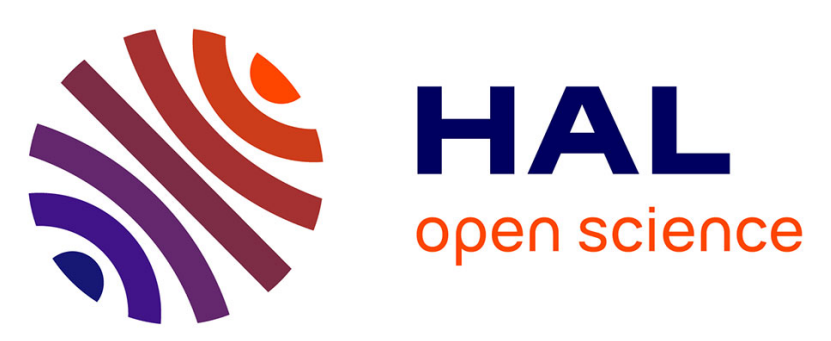

\title{
Characterization of polycrystalline materials using synchrotron X-ray imaging and diffraction techniques
}

Wolfgang Ludwig, A. King, M. Herbig, Peter Reischig, J.T. Marrow, Laurent Babout, E.M. Lauridsen, Henry Proudhon, Jean-Yves Buffiere

\section{- To cite this version:}

Wolfgang Ludwig, A. King, M. Herbig, Peter Reischig, J.T. Marrow, et al.. Characterization of polycrystalline materials using synchrotron X-ray imaging and diffraction techniques. JOM Journal of the Minerals, Metals and Materials Society, 2010, décembre, pp.22-28. 10.1007/s11837-010-0176-6 . hal-00534470

\author{
HAL Id: hal-00534470 \\ https://hal.science/hal-00534470
}

Submitted on 10 Nov 2010

HAL is a multi-disciplinary open access archive for the deposit and dissemination of scientific research documents, whether they are published or not. The documents may come from teaching and research institutions in France or abroad, or from public or private research centers.
L'archive ouverte pluridisciplinaire $\mathbf{H A L}$, est destinée au dépôt et à la diffusion de documents scientifiques de niveau recherche, publiés ou non, émanant des établissements d'enseignement et de recherche français ou étrangers, des laboratoires publics ou privés. 


\title{
Characterization of polycrystalline materials by combined use of synchrotron $\mathrm{X}$-ray imaging and diffraction techniques
}

\author{
W. Ludwig ${ }^{1,2}$, A. King ${ }^{3,4}$, M. Herbig ${ }^{1}$, P. Reischig ${ }^{2}$, J. Marrow ${ }^{3}$, L. Babout ${ }^{5}$, \\ E.M. Lauridsen ${ }^{6}$, H. Proudhon ${ }^{7},{ }^{J}$.Y. Buffière ${ }^{1}$
}

1) Université de Lyon, INSA-Lyon, MATEIS CNRS UMR 5510, 69621Villeurbanne, France

2) European Synchrotron Radiation Facility, BP220, 38043 Grenoble, France

3) School of Materials, University of Manchester, Manchester, M13 9PL, UK

4) GKSS research centre, Max-Planck-Str 1, 21502 Geesthacht, Germany

5) Computer Engineering Department, Technical University of Lodz, 90-924 Lódz, Poland

6) Institute for Sustainable Energy, Denmark Technical University, DK-4000 Roskilde, Denmark

7) MINES ParisTech, Centre des matériaux, CNRS UMR 7633, BP 87, 91003 Evry Cedex, France

The combination of synchrotron radiation X-ray imaging and diffraction techniques offers new possibilities for in-situ observation of deformation and damage mechanisms in the bulk of polycrystalline materials. Minute changes in electron density (i.e. cracks, porosities) can be detected using propagation based phase contrast imaging, a 3D imaging mode exploiting the coherence properties of third generation synchrotron beams. Furthermore, for some classes of polycrystalline materials, one may use a 3D variant of X-ray diffraction imaging, termed 'X-ray diffraction contrast tomography' (DCT). DCT provides access to the 3D shape, orientation and elastic strain state of the individual grains from polycrystalline sample volumes containing up to thousand grains. Combining both imaging modalities, one obtains a comprehensive description of the materials microstructure at the micrometer length scale. Repeated observation during (interrupted) mechanical tests provide unprecedented insight into crystallographic and grain microstructure related aspects of polycrystal deformation and degradation mechanisms.

\section{Introduction}

Since its introduction in the eighties of the past century, electron backscatter diffraction (EBSD) has enabled fundamental new insights into various aspects of the deformation and damage mechanisms in polycrystalline materials. The combination with in-situ observations in the scanning electron microscope and high resolution observations in the transmission electron microscope have contributed to our current understanding of the physical mechanisms involved in polycrystal deformation. More recently, it has been demonstrated that strain and rotation tensor components can be determined from electron backscatter patterns with an accuracy of $2 \cdot 10^{-4}$ Error! Reference source not found. However, despite progress in extending these techniques into the third dimension via (destructive) serial sectioning ${ }^{2}$ or ion beam milling ${ }^{3,4,5}$ electron microscopy lacks the capability of probing the temporal evolution of orientation and strain fields in the bulk of $\mu \mathrm{m}$ to $\mathrm{mm}$ sized specimen.

With progress in synchrotron X-ray imaging and diffraction techniques, this gap is currently about to be filled. X-ray beams can nowadays be focused to 100-200 nm spot size as a matter of routine ${ }^{6,7}$, line focus sizes of $30 \mathrm{~nm}$ have already been reported $^{8}$ and, at least from a theoretical point of view, down to nanometer spot sizes may be achievable with further progress in fabrication of X-ray optics ${ }^{9,10}$.

In conjunction with a 3D scanning technique termed differential aperture $\mathrm{x}$-ray microscopy $(\mathrm{DAXM})^{11}$, non-destructive 3D mapping with down to $200 \mathrm{~nm}$ spatial 
resolution $^{12}$ and an orientation and elastic strain resolution superior to the one obtained in scanning electron microscopy can be achieved. However, as any 3D scanning technique, DAXM can only provide limited time resolution, and typical analysis volumes are consequently small $\left(<100^{3}\right.$ voxels $)$.

In this paper we present a different approach to crystal microstructure characterization, based on monochromatic beam diffraction and imaging techniques. $3 \mathrm{D}$ X-ray diffraction microscopy ${ }^{13}$ (3D XRD) and related techniques ${ }^{14}$ benefit from faster data acquisition procedures, since either the entire sample volume or 2D sample cross-sections are illuminated, while scanning the sample around a single axis. The diffracted beams are acquired on a high resolution X-ray imaging detector and the use of polycrystal indexing ${ }^{15,14}$ and tomographic reconstruction approaches provide access to the 3D shape, position, orientation and elastic strain of the individual grains.

In the following we briefly review the principle, strength and limitations of a technique termed X-ray diffraction contrast tomograph ${ }^{16,17,14}$ which, in addition to 3D grain mapping enables simultaneous X-ray attenuation and/or phase contrast imaging. Like in electron microscopy, albeit at a different lengthscale, the conjunction of a imaging and diffraction mode on the same instrument can provide new insights into various aspects of polycrystal deformation and damage mechanisms. We finally discuss possible extensions to the currently established methodology.

\section{X-ray diffraction contrast tomography}

X-ray diffraction contrast tomography (DCT) is a variant of the previously introduced 3DXRD technique enabling simultaneous reconstruction of the 3D microstructure visible in $\mathrm{x}$-ray attenuation contrast and the 3D grain microstructure (shape and orientations) in suitable polycrystalline materials. The technique shares a common experimental set-up with conventional synchrotron radiation X-ray microtomography. In both cases, the sample is placed on a rotation stage and irradiated by an extended, parallel and monochromatic synchrotron X-ray beam. For the case of polycrystalline materials, each of the grains will pass through Bragg diffraction alignments multiple times during the sample rotation, producing diffracted beams. Beams diffracted at small angles will be captured on the detector system that covers an area substantially bigger than the sample (Figure 1). In the absence of significant orientation and strain gradients inside the grains, the diffracted beams form two-dimensional spots that can be treated as parallel projections of the diffracting grain. The analysis of Friedel pairs of these diffraction spots allows one to determine the crystallographic orientation and 3D shape of the grains in the sample. 

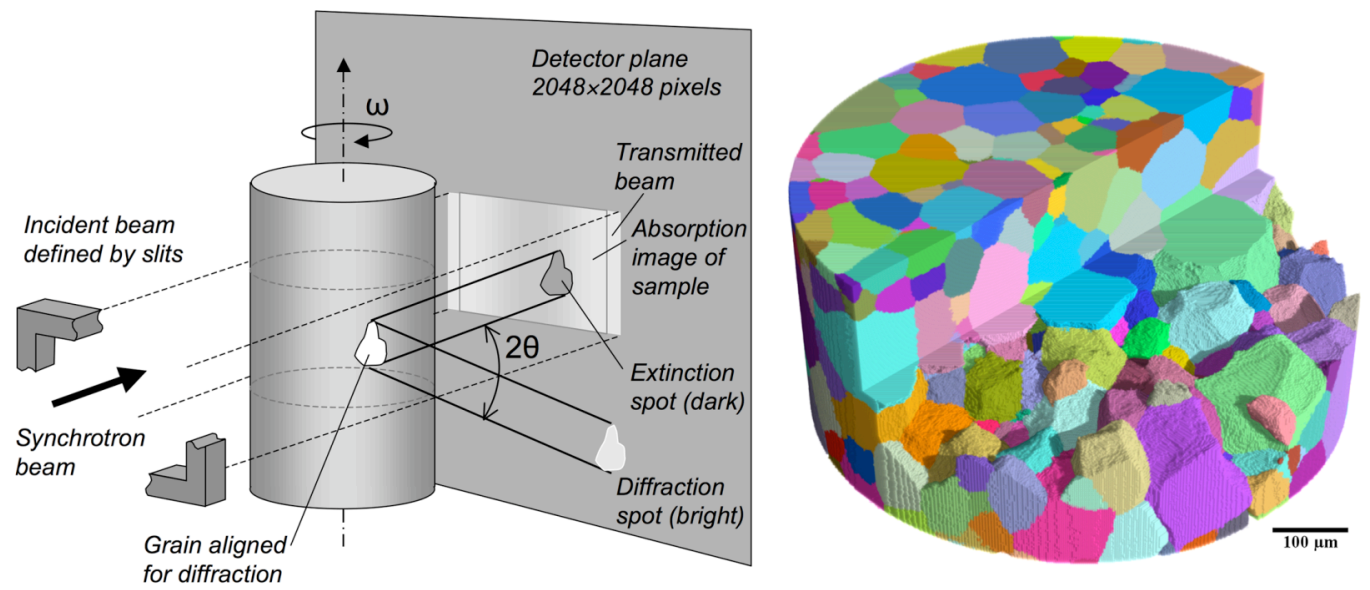

Figure 1: a) experimental setup for DCT, allowing for simultaneous acquisition of absorption and diffraction information by proximity of sample and detector. b) Reconstructed microstructure of a beta Ti sample containing 1000 grains.

The principal steps of the processing route are illustrated in Figure 2 and can be summarized as follows:

- Step 0: Acquistition. A stack containing several thousand combined diffraction images are acquired during continuous rotation movement of the sample over 360 degrees. The acquired volume may contain up to 100.000 diffraction 'blobs' (3D diffraction volumes), part of which may extend over several consecutive images

- Step 1: Segmentation. Consecutive images of the 3D diffraction 'blobs' are summed and segmented into 2D diffraction spots using thresholding techniques. Information about the spots is stored in a database (center of mass position, intensity, area, etc..)

- $\quad$ Step 2: Pair Matching. From axial symmetry consideration a grain which diffracts for an angular position $\omega$ diffracts at $\omega+180^{\circ}$ (hkl and -h-k-l reflection). These so-called Friedel pairs of diffraction spots are detected automatically using a combination of spatial and crystallographic criteria. Once a pair of spots is detected, the diffraction angles describing the geometry of the diffraction event (plane normal, scattering vector) can be calculated.

- Step 3: Indexing. The detected Friedel pairs are sorted into sets belonging to the same grain ("indexing"). This is done by checking both spatial and crystallographic consistency criteria. The diffracted beams arising from a grain have to intersect at the grain position, and the angle between scattering vectors has to reflect the crystal symmetry. Typically several tens of diffraction spots can be identified per grain and the (average) grain orientation and elastic strain tensor can be determined.

- Step 4: Grain Reconstruction. In the absence of strong orientation and strain gradients within a grain, the diffraction spots can be considered as parallel projections of the grain from which they arise. These projections are used to reconstruct the three-dimensional grain shape using algebraic reconstruction techniques $(\mathrm{ART})^{18}$. This algorithm allows the reconstruction of $3 \mathrm{D}$ shapes from a limited number of projections. Each grain is reconstructed individually. The assembly of all the reconstructed grains produces the 3D grain microstructure of the sample. 
- Step 5: Absorption contrast tomogram The direct beam projection images recorded during the scan are used to reconstruct the absorption contrast tomogram of the sample by conventional filtered backprojection reconstruction. The 3D tomogram obtained can be superimposed on the 3D grain map determined by DCT.

\section{Strengths and Limitations}

X-ray diffraction contrast tomography is ideally suited for the characterization of mono (or dual) phase polycrystalline materials fulfilling some conditions on grain size, microtexture, intragranular orientation and elastic strain gradients. The extended beam (2D) illumination mode and the use of tomographic reconstruction schemes results in a tremendous gain in time resolution: when performed at a high flux beamline and using state of the art detector technology, a full scan, comprising typically 3600 images and covering a volume of $\sim 400^{3}$ voxels (up to 1000 grains) can be acquired in less than 10 min (compared to 3 months when using a 3D scanning approach). On the other hand the ultimate spatial resolution is limited by current Xray imaging detector technology ${ }^{19}$ to values of about $1 \mu \mathrm{m}$ and the analysis route outlined in the previous section does not provide access to local values of orientation and elastic strain inside individual grains (possible extensions of the technique in this direction are under investigation ${ }^{20}$ ). Approximating the diffraction spots as parallel projections of the $3 \mathrm{D}$ grain volume is only valid in the case of weak intragranular

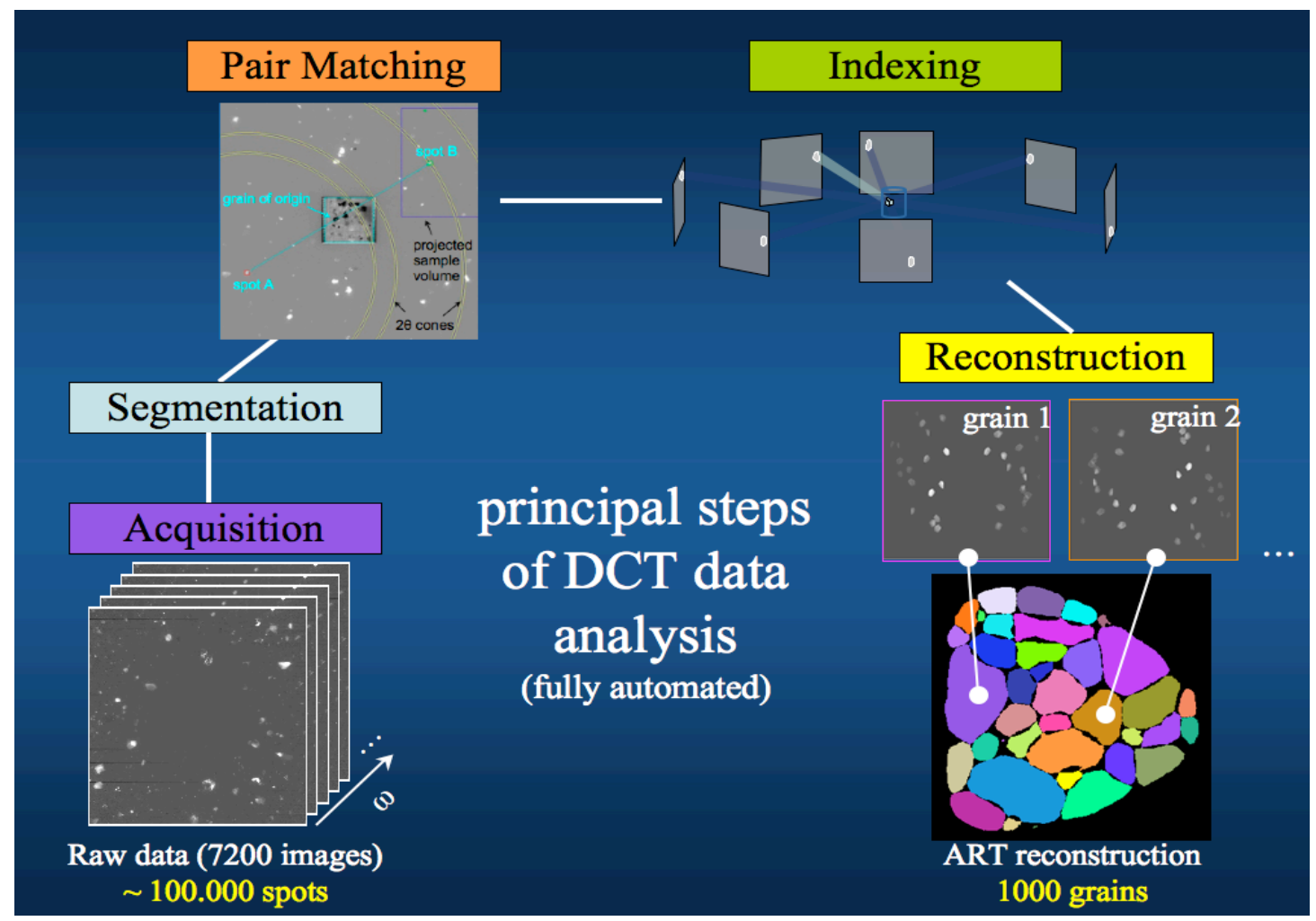

Figure 2: Schematic diagram of the $3 D$ grain reconstruction process employed in diffraction contrast tomography. Acquisition times range from several minutes to several hours - current reconstruction time is about one day. 
orientation and elastic strain gradients - and the accuracy of reconstructions based on this assumption will degrade with increasing levels of plastic deformation of the material. For strain values beyond a few percent, different reconstruction schemes, based on forward simulation schemes ${ }^{21,22}$ seem more appropriate. Changing to 1D (line beam) illumination mode further simplifies the interpretation of the diffraction data, but necessarily compromises the ultimate time resolution.

\section{Application examples}

In the following we will showcase in how far the combined imaging and diffraction technique can provide insight into damage mechanisms, encountered in structural materials under service conditions: (1) propagation of short fatigue cracks ${ }^{23}$ and (2) stress corrosion cracking in an austenitic stainless steel ${ }^{24}$. In both cases the $3 \mathrm{D}$ grain microstructure of the material has been determined by means of X-ray diffraction contrast tomography in its initial state, before the onset of damage. Next, the temporal evolution of damage has been observed by interrupted in-situ observations using high resolution phase contrast tomography and dedicated mechanical testing equipment, directly installed on the tomographic imaging set-up. The third example reports on work in progress, addressing the characterization of type II stresses in a grain mapped polcrystal and possible coupling with crystal plasticity finite element simulations on the real microstructure.

\section{$\underline{\text { Investigation of crystallographic aspects of short fatigue crack propagation }}$}

The propagation of short fatigue cracks is governed, in combination with the externally applied stress field, by the microstructure of the material ${ }^{25}$. Surface observations by SEM and EBSD indicate that cracks may propagate on crystallographic slip planes during the initial growth stage (stage I) ${ }^{26}$. However, with electron microscopy, the propagation can only be observed on a $2 \mathrm{D}$ surface section whereas the crack itself is clearly $3 \mathrm{D}$ in nature. Access to the $3 \mathrm{D}$ crack shape and facet orientation may be obtained post-mortem by serial sectioning techniques ${ }^{27}$ - but one still lacks the history of the $3 \mathrm{D}$ propagation process. The combination of X-ray diffraction contrast tomography and high-resolution phase contrast tomography is a powerful way to gain insight into crystallographic aspects of the $3 \mathrm{D}$ propagation process. Figures 3a-c show three out of a total of 26 time steps recorded during propagation of a short fatigue crack in a metastable beta-Ti alloy. The same crack after 75,000 cycles is shown with its surrounding grains in figure 3d. After segmentation and transformation of the $3 \mathrm{D}$ crack data into a surface mesh one may associate a data structure to each of the surface elements, containing information about the local orientation and the number of fatigue cycles at which the area was swept by the crack front. Quantitative evaluation of local crack growth rates as well as global and local analysis of the fracture surface orientation can be carried out by interrogation of this data structure ${ }^{23}$. The analysis of fracture surface orientation is illustrated in Figure 3e, where the local orientation is colour coded with respect to the orientation of the corresponding grain. Closer inspection of crack propagation in selected grains shows clear signs of crystallographic propagation. This is illustrated in Figure $3 \mathrm{f}-\mathrm{g}$, showing the 5 successive crack front locations recorded during crack 
propagation in the grain highlighted in Figure 3e. Here the crack is observed to accelerate as soon as it changes its growth plane to a well defined crystallographic (101) plane. However, for the alloy system and the spatial resolution employed in this study ( $\sim 2 \mu \mathrm{m}$ full width at half maximum of the detector point spread function), one can not observe a global prevalence of orientations corresponding to reported slip planes in body centered cubic metals $\left(\{110\},\{112\},\{123\}^{28}\right)$. The same experiment, carried out in a different beta $\mathrm{Ti}$ alloy system shows almost exclusive propagation in single slip mode ${ }^{29}$. One may therefore speculate, if the absence of crystallographic signature indicates a lack of resolution to resolve microscopic facets caused by alternating slip on two simultaneously activated slip systems ${ }^{30}$. This point will be addressed in future work which will include 3D inspection of the fracture surface at higher spatial resolution, as provided by X-ray zoom tomography ${ }^{31}$.
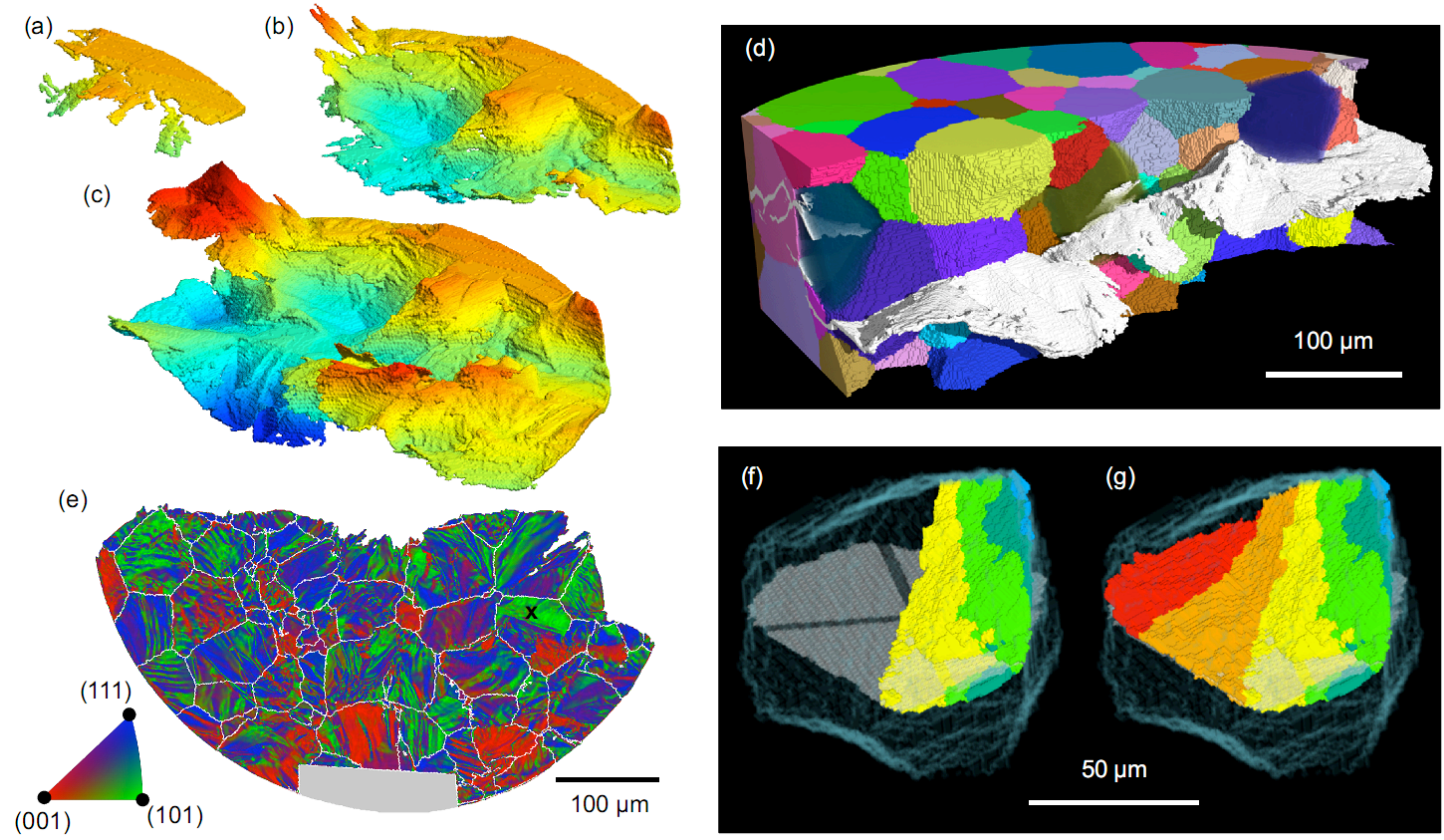

Figure 3: a-c) 3D rendition of a fatigue crack in a Ti alloy reconstructed from phase contrast tomography after 46, 61 and $75 * 10^{3}$ loading cycles. The color corresponds to the vertical crack position. d) $3 D$ rendition of the combined data set showing the crack and the surrounding grains. e) Surface mesh representing the fracture surface, colour coded with respect to its crystallographic orientation. Grain boundaries are labeled in white. f,g) Crack propagation within the grain labeled in Fig. 3e. The activated (110) slip plane and the two $<111>$ slip directions are highlighted in gray and black, respectively.

Investigation of intergranular stress corrosion cracking in stainless steel

Intergranular stress corrosion cracking in stainless steels is a damage mechanism whereby in the presence of an applied load, cracks propagate along sensitised grain boundaries by localised corrosion. The grain boundary sensitisation can occur in stainless steels due to localised depletion of chromium. This can occur due to thermal processing, such as in the heat affected zone around a weld, but it can also occur due to irradiation ${ }^{32,33}$. As a result, under certain circumstances IGSCC may develop in the 
cooling circuits of light water reactors ${ }^{34}$. The susceptibility of individual grain boundaries to sensitisation is related to their 3D geometry. Both the crystallographic orientations of adjoining grains, as well as the plane of the grain boundary affect grain boundary properties. The coincident site lattice notation which is often used to categorize grain boundaries considers only the grain orientations, and neglects the influence of the boundary plane ${ }^{35}$. Certain grain boundaries have been shown to resist IGSCC, forming bridging across propagating cracks ${ }^{36}$. The resistance of a material to IGSCC is therefore related to the 3D network of grain boundaries, and researchers have attempted to model this behaviour ${ }^{37}$.

The ability of DCT to provide complete 3D grain boundary descriptions of all the boundaries in a sample, combined its non-destructive nature, make DCT an ideal tool for the study of IGSCC. This has been undertaken in 302 stainless steel with thermally sensitised grain boundaries ${ }^{24}$. The grain structure of the sample was first mapped using DCT. This reveals grain shapes and orientations, and statistics describing the population of grain boundaries. In-situ observations of IGSCC propagation were then made using synchrotron microtomography. Using a specially designed in-situ apparatus, the sample was placed in a solution of acidified potassium tetrathionate $\left(\mathrm{K}_{2} \mathrm{~S}_{4} \mathrm{O}_{6}\right)$, and loaded to initiate cracking. At intervals the load was removed, and tomograms were recorded to show the 3D shape of the advancing crack. By correlating these tomograms with the DCT dataset, those boundaries that crack can be separated from the general population. Furthermore, because of the temporal information available from the multiple time steps, those boundaries which form bridges across the advancing crack can also be identified, and their properties investigated. Branches of the crack below the final fracture surface are also accessible. Figure $4 \mathrm{a}$ shows all grain boundaries in a section through the sample, overlaid with the crack path, highlighting those boundaries which have been associated with the crack. Due to the crack opening displacements a correction must be applied to the crack tomograms before they can be aligned with the DCT grain boundaries. This has been performed using a routine based on pose estimation, described in $^{38}$. Despite this, some distortions remain and can be perceived in Figure 4. Those grain boundaries associated with the crack, and those which formed bridges, are coloured according to the legend. Bridges have been extracted from the volumetric image using a hole filling algorithm ${ }^{39}$, as they are equivalent to holes in the object that corresponds to the crack, and therefore cannot be segmented using classical methods such as histogram-based segmentation. Figure $4 \mathrm{~b}$ shows the same boundaries coloured according to the coincident site notation, with low $\Sigma$ boundaries highlighted. In the example shown it can be seen that the crack propagates on nonlow $\Sigma$ boundaries. The specific geometrical properties of the bridging grain boundaries can be investigated. Comparing Figures $4 a$ and $4 b$ it can be seen that in some cases the bridging grain boundaries appear to be associated with the presence of twinning in a grain, although they are not themselves low $\Sigma$ boundaries. In a previous investigation of this type, three ligaments remaining after the propagation of the IGSCC were found to be a low angle grain boundary, a low $\Sigma$ boundary $(\Sigma 11)$, and a boundary, the plane of which was orientated close to low $\{\mathrm{hkl}\}$ index crystallographic planes in both the adjoining grains ${ }^{24}$. By including those ligaments that form during the propagation of the crack, but which subsequently fracture, the number of ligaments investigated can be increased. Figure 5a is a $3 \mathrm{D}$ rendering of the grains in one half of the fractured specimen. Figure $5 \mathrm{~b}$ show the same surface, but coloured using the same key as figure 4a to show the temporal evolution of the crack, and the location of bridging ligaments. This can be used as a complement to post-mortem 
SEM imaging of the fracture surface, adding temporal information to conventional fractography.

A limitation of the current analysis is that each grain boundary facet is considered as a single entity. The grain boundary plane is found by fitting a plane to all voxels associated with the facet. Thus each facet is described by the misorientation of the adjoining grains, the average boundary plane, and the area of the boundary. However, for non-planar grain boundaries, a more sophisticated analysis considering the local grain boundary plane is required. This limitation will be addressed in future work, enabling the analysis to better address the situation that part of a boundary forms a bridge in the advancing crack.

(a)

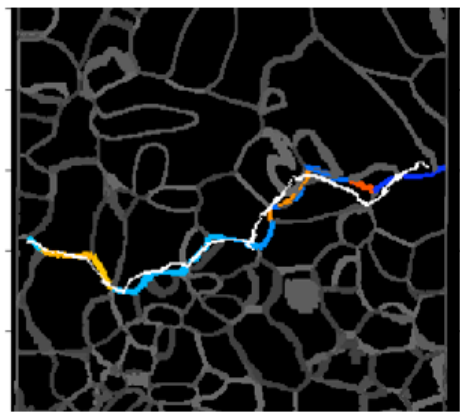

\begin{tabular}{ll|l} 
step 1: & crack & bridge \\
step 2: & crack & bridge \\
step 3: & crack & bridge \\
step 4: & crack & bridge \\
step 5: & crack & bridge \\
step 6: & crack & bridge \\
step 7: & crack & bridge
\end{tabular}

(b)
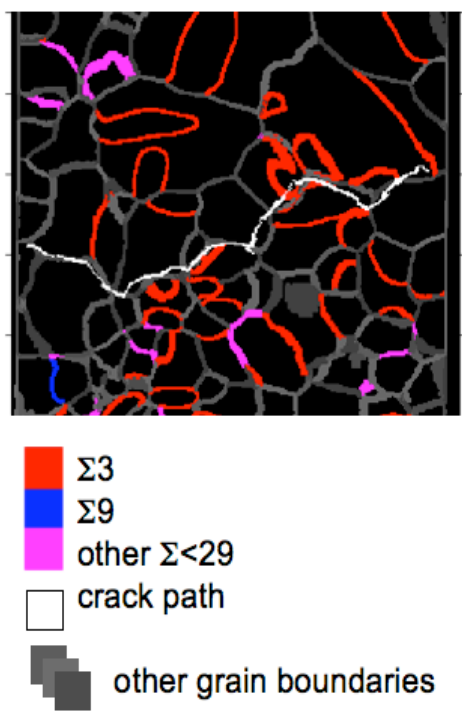

Figure 4: a) Cross-sectional view of stress-corrosion sample with the crack path highlighted in white, and the cracked boundaries and crack bridges at different propagation steps colored in red and blue, respectively. b) Overlay of crack path with the grain boundary network, color coded with respect to the coincident lattice notation.

(a)

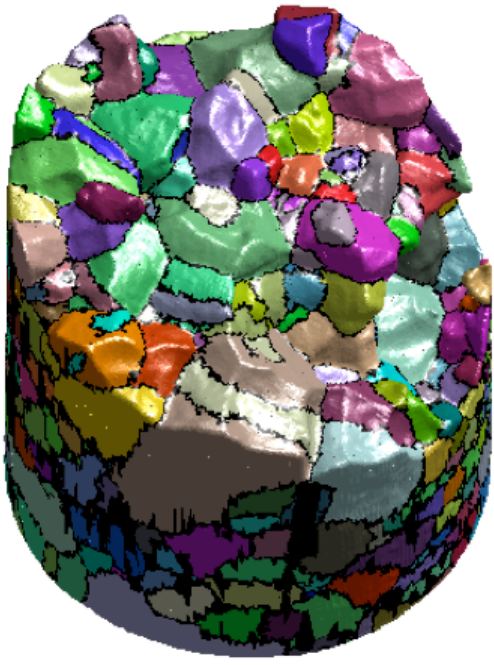

(b)

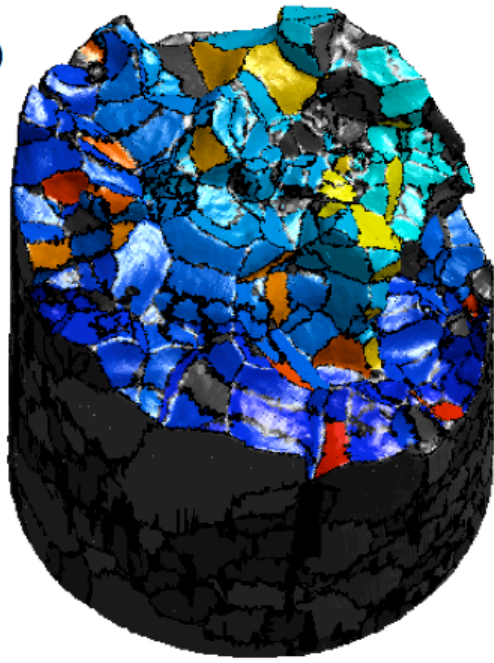


Figure 5: 3D surface renderings of the grains from one half of the sample after IGSCC. Surfaces colored according to a) grain, b) cracked boundaries and crack bridges at each propagation step (as figure 4a)

$\underline{\text { Characterization of elastic deformation and image based finite element simulation }}$

Despite the fact that the near field detection geometry used in diffraction contrast tomography does not provide optimum angular accuracy ${ }^{1}$, a feasibility study ${ }^{40}$ on a large grained Ti polycrystal indicates that grain average elastic strain tensors can be extracted with a resolution of about $5 \cdot 10^{-4}$. The dogbone shaped tensile test sample was mounted in a miniature tensile test rig and two X-ray diffraction contrast tomography acquisitions were performed at 18 and $450 \mathrm{MPa}$ of applied stress. Figure $6 \mathrm{c}$ shows a 3D rendition of the material with part of the grains set to transparent. The alloy system used in this study offers an elegant way for quantitative evaluation of the accuracy of the grain map: a specific heat treatment leads to layer-like precipitation of alpha $\mathrm{Ti}$ (hcp) and segregation of alloying elements along the beta grain boundary network, which in turn can be imaged by phase contrast tomography ${ }^{41,42,43,44}$. Figure $6 \mathrm{a}$ and $\mathrm{b}$ show the same cross-section trough the sample as determined by diffraction contrast (before heat treatment) and phase contrast imaging, respectively. Apart from excellent correspondence one may note the absence of alpha phase decoration on one of the grain boundaries (see arrow in Figure 6b). The two grains forming this grain boundary are tilted by a small angle ( 5.4 degrees) with respect to each other. The missing boundary corresponds therefore to a low angle boundary, associated with a low grain boundary energy and hence reduced driving force for wetting by a second phase.
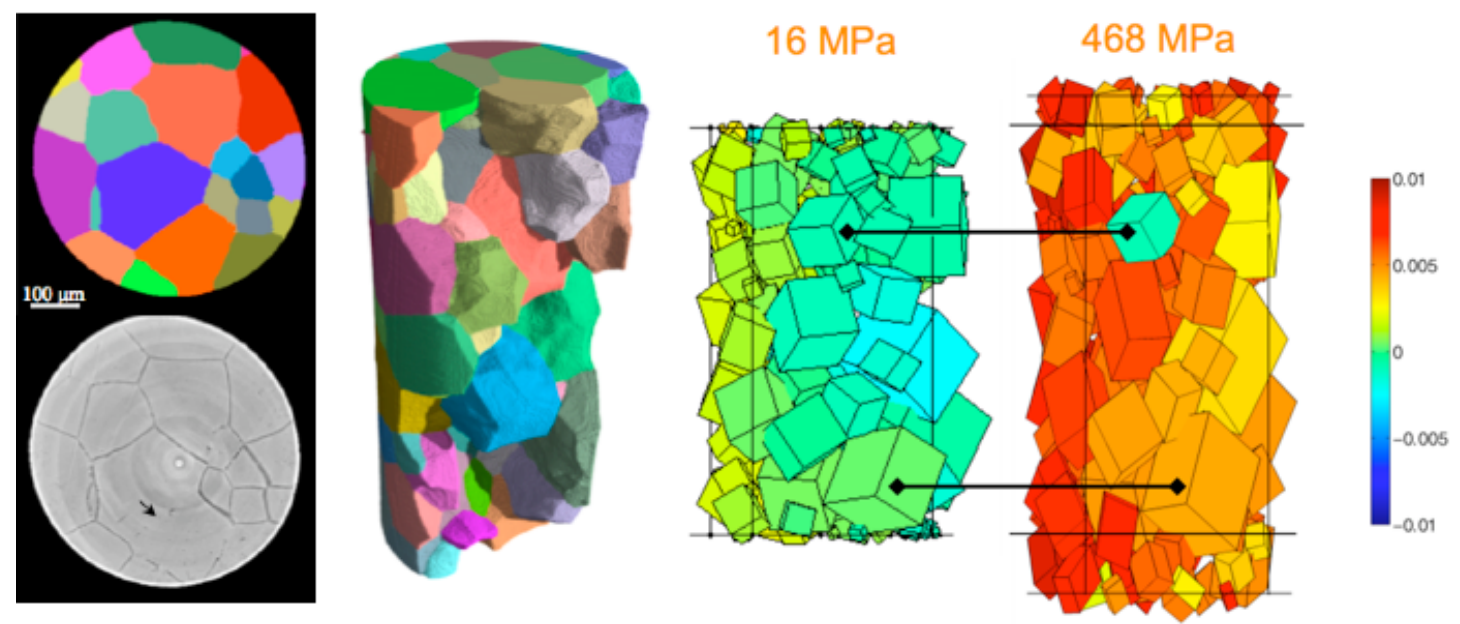

Figure 6: a) sample cross-section as determined by diffraction contrast tomography b) corresponding cross-section as observed with phase contrast tomography c) $3 D$

\footnotetext{
${ }^{1}$ In contrast to a low spatial resolution diffraction detector positioned in the far field, the diffraction angles determined from a high resolution detector are affected by drifts and/or mechanical inaccuracies of the instrument in the micrometer range.
} 
rendering of the sample with part of the grains set to transparent d) parallelepiped representation of the deformed grains, colour coding according to axial strain component $\left(\varepsilon_{33}\right)$.

The 6 components of the (grain average), elastic strain tensors were determined via a maximum likelihood fitting procedure, minimizing the residual between experimentally observed and expected spot positions. Figure $6 \mathrm{~d}$ shows a $3 \mathrm{D}$ view of the sample volume with individual grains represented as parallelepipeds rotated and deformed according to the orientation and deformation state as determined from DCT. At both levels of applied load, one can observe a gradient in the axial strain component $\varepsilon_{33}$ perpendicular to the axis of the cylindrical sample. This indicates nonuniaxial loading conditions which might be attributed to misalignments and/or deviations from the nominal sample / crosshead geometry. Further improvement of strain sensitivity has been reported ${ }^{45}$ by including a larger number of diffraction events when acquiring data on a conventional diffraction detector system with larger field of view and pixel size, thereby also decreasing the sensitivity with respect mechanical inaccuracies of the instrument.

\section{Perspectives for future work}

Giving access to the position and orientation of individual grains in the bulk of a polycrystalline specimen, the outlined methodology offers the possibility to apply complementary measurement techniques on selected grains, with known 3D grain neighbourhood. For instance, one may align a specific reflection of the grain of interest and perform reciprocal space mapping ${ }^{46}$ or X-ray line profile measurements ${ }^{47}$ on a detector positioned in the far field. One may also consider switching from extended to focused beam, micro-scanning diffraction mode for high spatial resolution mapping of local orientation and elastic strain fields inside the grain of interest.

For studies extending to large values of plastic strain, one may consider mapping of an undeformed microstructure containing a fine dispersion of internal markers (such as porosities, precipitates or inclusions) by means of DCT and subsequent characterization of the deformation gradient tensor field by means of particle tracking or digital volume correlation techniques ${ }^{48,49}$.

Last but not least we mention ongoing efforts to simulate and match the experimentally observed deformation behaviour with crystal plasticity simulations. The data obtained from a DCT scan of a polycrystalline sample can be turned into a finite element mesh. The best results can be expected with tetrahedron free meshing which preserves the grain boundary morphology (Figure 7a). Provided the single crystal elastic constants and constitutive law of the material are known, crystal plasticity computations can be carried out to retrieve the strain heterogeneities within the grains. The grain average values can in principle be compared directly with the elastic strain tensors obtained from diffraction measurements. In practice, uncertainties in the loading conditions may complicate such direct comparison.

The quantitative analysis of damage mechanisms like fatigue or stress corrosion cracking requires computation of the stress and strain fields in the vicinity of the crack tip. The comparison of experimentally observed and simulated material behaviour will provide guidance what kind of models (cohesive zones, damaging elements or even coupling with discrete dislocation dynamics) can best account for 
the observed cracking behaviour.

An example with idealized crack geometry is showed on Fig. $7 \mathrm{~b}$ for illustration purpose. A flat penny shape crack has been inserted into the beta titanium microstructure of Fig 6c. The grain topology has been perfectly preserved and the mesh is refined around the crack to capture the stress concentration. Efforts are underway to tackle the more complex meshing problem of inserting real crack morphologies (Fig. 3a) into the 3D grain network.

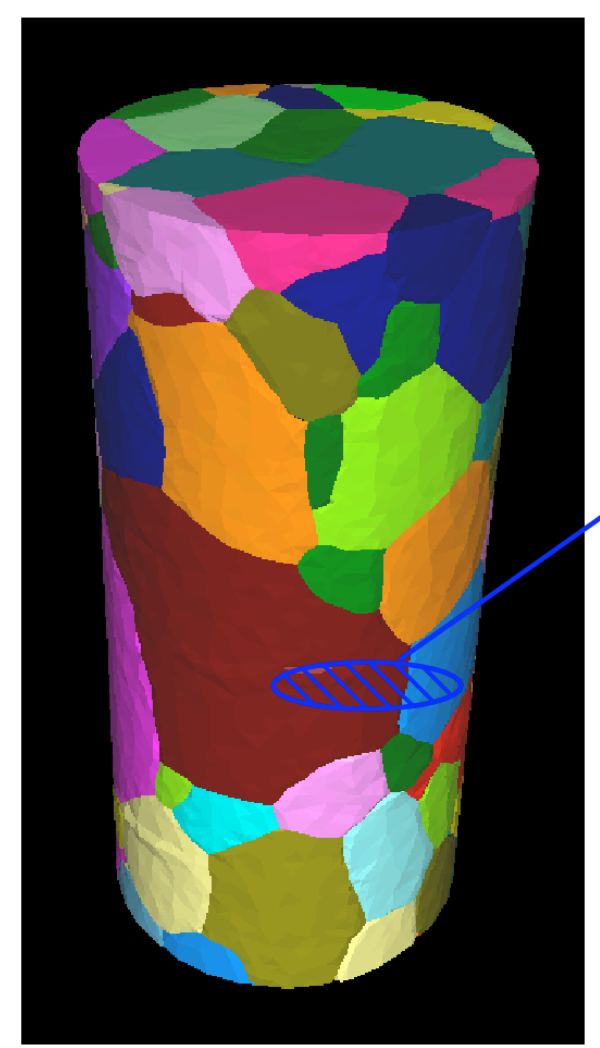

polycrystal from DCT

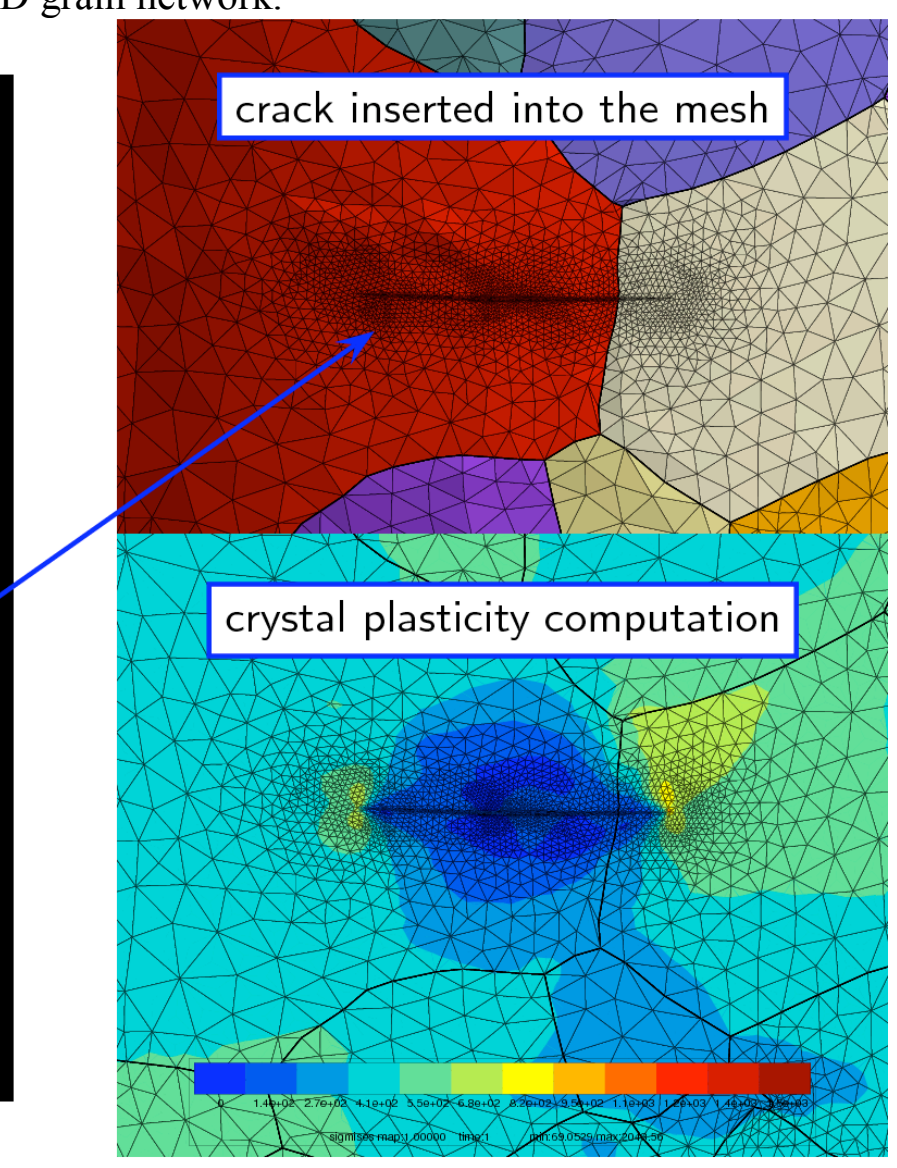

meshed with tetrahedrons

Figure 7: a) Finite element mesh representation the sample depicted in Figure 5c. b) Insertion of penny shaped crack and mesh refinement in proximity of the crack tip c) von Mises stress calculated by crystal plasticity finite element computation

\section{Conclusions}

Synchrotron radiation X-ray imaging and diffraction techniques can characterize the 3D grain microstructure non-destructively and open new possibilities for investigation of deformation and damage mechanisms in the bulk of polycrystalline materials. The current paper has focused on X-ray diffraction contrast tomography, a technique combining the principles X-ray diffraction imaging (topography) and image reconstruction from projections (computed tomography). This variant of $3 \mathrm{D}$ grain mapping describes the grain microstructure in terms of average grain orientation and elastic strain values and applies to large grained mono (or dual-) phase materials and 
works best for materials displaying limited levels of intragranular orientation spread and/or grain-substructures.

The technique shares a common experimental setup with X-ray microtomography and can be easily combined with phase sensitive imaging techniques. The combined characterization provides quantitative, comprehensive description of the material's microstructure at the micrometer lengthscale, in terms of 3D grain shape, orientation, local attenuation and/or electron density distribution. The produced 3D grain maps can be used as input for crystal plasticity simulations. Efforts are underway to extend the technique towards characterization of local orientation and elastic strain fields. The knowledge of the position, shape and orientation of bulk grains enables complementary, single crystal characterization techniques to be applied on a sub-set of grains. This in turn offers potential for comparison between experimentally observed and numerically simulated deformation behaviour and will provide guidance for improvement of the models these simulations are based on.

\section{Acknowledgements}

The authors gratefully acknowledge ESRF and staff from beamlines ID19 and ID11 for provision of beamtime and support during the experiments. W.L. and E.M.L acknowledge the Danish National Research Foundation for supporting the Center for Fundamental Research 'Metal structures in 4D'. A.K. acknowledges funding received from the Engineering and Physical Sciences Research Council, UK. L.B. acknowledges 6 months spent at the University of Manchester in the framework of the DENIDIA project (FP6-Marie Curie ToK-Dev scheme. Contract no.: MTKDCT-2006-039546).

\section{References}

1. A. J. Wilkinson, G. Meaden, and D. J. Dingley.. Ultramicroscopy, 106(4-5):307 - 313, 2006.

2. D. J. Rowenhorst, A. Gupta, C. R. Feng, and G. Spanos, Scripta Materialia, 55(1):11-16, 2006

3. T. Matteson, S. Schwarz, E. Houge, B. Kempshall, and L. Giannuzzi Journal of Electronic Materials, 31(1):33-39, 2002.

4. S. Zaefferer, S. Wright, and D. Raabe. Microscopy and Microanalysis, 13, 1508-1509, 2007.

5. Y. Bhandari, S. Sarkar, M. Groeber, M. D. Uchic, D. M. Dimiduk, and S. Ghosh. Computational Materials Science, 41(2):222-235, 2007.

6. O. Hignette, P. Cloetens, G. Rostaing, P. Bernard, and C. Morawe, Review of Scientific Instruments, 76(6):063709-5, 062005.

7. W. Liu, G. Ice, J. Tischler, A. Khounsary, C. Liu, L. Assoufid, and A. Macrander. Review of Scientific Instruments, 76: 2005

8. H. C. Kang, J. Maser, G. B. Stephenson, C. Liu, R. Conley, A. T. Macrander, and S. Vogt. Physical Review Letters, 96(12), 032006

9. C. G. Schroer. Physical Review B, 74(3), 072006.

10. C. G. Schroer and B. Lengeler. Physical Review Letters, 94(5), 022005

11. B. C. Larson, W. Yang, G. E. Ice, J. D. Budai, and J. Z. Tischler. Nature, 415(6874):887890,2002

12. G. E. Ice, J. W. L. Pang, B. C. Larson, J. D. Budai, J. Z. Tischler, J.-Y. Choi,W. Liu, C. Liu, L. Assoufid, D. Shu, and A. Khounsary. Materials Science and Engineering: A, 524(1-2):3-9, 2009.

13. H. F. Poulsen. Three-dimensional X-ray diffraction microscopy. Springer, 2004. 
14. W. Ludwig, P. Reischig, A. King, M. Herbig, E. M. Lauridsen, G. Johnson, T. J. Marrow, and J. Y. Buffiere.. Review of Scientific Instruments, 80(3):033905-9, 2009

15. E. M. Lauridsen, S. Schmidt, R. M. Suter, and H. F. Poulsen. Journal of Applied Crystallography, 34(6):744-750, Dec 2001.

16. G. Johnson, A. King, M. G. Honnicke, J. Marrow, and W. Ludwig. Journal of Applied Crystallography, 41:310-318, 2008

17. W. Ludwig, S. Schmidt, E. M. Lauridsen, and H. F. Poulsen. J. Appl. Cryst. 41 (2008), pp 302-309

18. R. Gordon, R. Bender, and G. T. Herman, Journal of Theoretical Biology, 29(3):471 481,1970

19. T. Martin and A. Koch, J. Synchrotron Rad., 13:172-179, 2006.

20. A. King, P. Reischig, S. Martin, J. Fonseca, M. Preuss, and W. Ludwig. 31st Risø International Symposium on Materials Science: Challenges in materials science and possibilities in $3 D$ and $4 D$ characterization techniques, volume 31, 2010

21. R. M. Suter, D. Hennessy, C. Xiao, and U. Lienert. Review of Scientific Instruments, 77(12):123905, 2006.

22. S. West, S. Schmidt, H. Sorensen, G. Winther, H. Poulsen, L. Margulies, C. Gundlach, and D. J. Jensen. Scripta Materialia, 61(9):875 - 878, 2009.

23. M. Herbig, A. King, P. Reischig, J.Y. Buffière, E.M. Lauridsen, T.J. Marrow, W. Ludwig, Acta Mater., accepted.

24. A. King, G. Johnson, D. Engelberg, W. Ludwig, and J. Marrow, Science, 321(5887):382385,2008

25. D. Taylor, J.F. Knott, Fatigue of Engineering Materials and Structures, 4(2):147-155, 1981.

26. O. Dueber, B. Kuenkler, U. Krupp, H.-J. Christ, C.-P. Fritzen, Int J Fatigue 28: 983992, 2006.

27. P. Clément, J.P. Angéli, A. Pineau, Fatigue Fract Eng M 7(4) :251, 1984.

28. Honeycombe RWK. Plastic Deformation of Metals, second sub ed. Edward Arnold; 1984.

29. M. Herbig, A. King, H. Proudhon, J. Buffière, and W. Ludwig. unpublished.

30. P. Neumann, Acta Met 17(9):1219, 1969.

31. R. Mokso, P. Cloetens, E. Maire, W. Ludwig, and J.-Y. Buffière.. Applied Physics Letters, 90(14):144104, 2007.

32. S.M. Bruemmer, G.S. Was, J. Nucl. Mater. 216 (1994) 348

33. P. Scott, J. Nucl. Mater. 211 (1994) 101

34. P.M. Scott, Corrosion 56 (2000) p771

35. V. Randle, Acta Mater. 46 (1998) 1459

36. L. Babout, T.J. Marrow, D. Engelberg, P.J. Withers, Mater. Sci. Technol. 22 (2006) 1068

37. A.P. Jivkov, N.P.C. Stevens, T.J. Marrow, Comput. Mater. Sci. 38 (2006) 442

38. L. Babout, M. Janaszewski, T.J. Marrow, P.J. Withers. Unpublished.

39. M. Janaszewski, M. Couprie ,L. Babout, Pattern Recogn., 43:3548-3559, 2010.

40. P. Reischig. Determination of elastic strain tensors from x-ray diffraction contrast tomography. Master's thesis, Delft University of Technology, 2008.

41. P. Cloetens, M. Pateyron-Salomé, J. Y. Buffière, G. Peix, J. Baruchel, F. Peyrin, M. Schlenker, J. Appl. Phys. 81, 5878 (1997)

42. P. Cloetens, W. Ludwig, J. Baruchel, D. Van Dyck, J. Van Landuyt, J. P. Guigay, and M. Schlenker, Appl. Phys. Lett., 75:2912-2914, 1999

43. S. Dey, E. M. Lauridsen, W. Ludwig, D. J. Rowenhorst, and R. Fonda., Proceedings of the Titanium 2007 Conference

44. W. Ludwig, A. King, P. Reischig, M. Herbig, E. M. Lauridsen, S. Schmidt, H. Proudhon, S. Forest, P. Cloetens, S. R. d. Roscoat, J. Y. Buffière, T. Marrow, and H. F. Poulsen. Materials Science and Engineering: A, 524:69-76, 2009.

45. J. Oddershede, S. Schmidt, H. F. Poulsen, H. O. Sørensen, J. Wright, and W. Reimers. Journal of Applied Crystallography, 43(3):539-549, Jun 2010.

46. B. Jakobsen, H. F. Poulsen, U. Lienert, J. Almer, S. D. Shastri, H. O. Sorensen, C. 
Gundlach, and W. Pantleon. Science, 312(5775):889-892, 52006.

47. T. Ungar, G. Ribarik, L. Balogh, A. Salem, S. L. Semiatin, and G. Vaughan, Scripta Materialia, 63:69-72, 2010.

48. B. Bay. Journal of Strain Analysis for Engineering Design, 43:745-760, 2008

49. J. Rannou, N. Limodin, J. Réthoré, A. Gravouil, W. Ludwig, M. Baïetto-Dubourg, J. Buffière, A. Combescure, F. Hild, and S. Roux. Computer Methods in Applied Mechanics and Engineering, 199:1307-1325, 2010. 